seasons. Increased staffing of credentialed sports medicine clinicians at domestic and international events to recognize and document mTBI.

Main Outcome Measurements Improved documentation of mTBI to influence prevention, care, and return to play interventions.

Results Data identified two primary mechanisms of injury leading to diagnosed mTBI: indirect head trauma from a blow to the mask from an opponent's weapon guard and falling backwards onto the strip causing direct posterior trauma to the head. The acceleration-deceleration mechanism of injury (whiplash) was also a component in both of these identified injury mechanisms.

Conclusions Increased clinician staffing at domestic and international events has directly correlated with an increase in documentation and overall mTBI prevention, diagnosis, care, and return to play considerations.

\section{CURRENT PRACTICE FOR SAFE RETURN-TO-PLAY AFTER LATERAL ANKLE SPRAIN: A SURVEY AMONG FRENCH- SPEAKING PHYSICIANS}

${ }^{1}$ Aude Aguilaniu, ${ }^{1}$ Jean-Louis Croisier, ${ }^{3}$ Cédric Schwartz, ${ }^{2}$ Nadia Dardenne, ${ }^{5}$ Pieter D'Hooghe, ${ }^{4}$ Yanis Zahraoui, ${ }^{1}$ Romain Collin, ${ }^{1}$ Jean-François Kaux, ${ }^{1,6}$ Géraldine Martens. 'Department of Sport Sciences of University of Liège, Liège, Belgium; ${ }^{2}$ Département of public health of science of University of Liège, Liège, Belgium; ${ }^{3}$ Laboratory of Human Motion Analysis, Faculty of Applied Sciences, University of Liege, Liège, Belgium; ${ }^{4}$ Physiotherapist, Liège, Belgium; ${ }^{5}$ Chief of Surgery at Aspetar Hospital, Doha, Qatar; ${ }^{6}$ ReFORM IOC Research Centre, Liège, Belgium

\subsection{6/bjsports-2021-IOC.97}

Background The incidence of lateral ankle sprain (LAS) in sports and risk of LAS re-injury is high.

Objective The purpose of this study was to see how physicians use existing recommendations in the literature to decide a safe return-to-play (RTP) after a LAS. We hypothesize that physicians are using the published recommendations to make this RTP decision.

Design An online survey was designed to identify clinical practice patterns to decide a RTP decision after a LAS.

Setting The physicians were contacted by e-mail containing an electronic link for survey access between January and February 2019. They were instructed to answer all questions that related to their clinical practice of patients after a LAS.

Patients (or Participants) 109 physicians answered the entire survey. Among the 109 physicians, 59 had a sport education and 50 had no specific sport education.

Interventions (or Assessment of Risk Factors) Physicians selected the criteria and details on the assessment they used in daily practice for RTP decision after a LAS.

Main Outcome Measurements Physicians should select a maximum of 5 items among the 9 suggested: 'pain, swelling, ankle range of motion, ankle muscle strength, feeling of instability, proprioception, ability to do functional tasks and other criteria'.

Results Pain, functional and instability criteria are the most selected by overall physicians. Sport physicians (94\%) selected significantly more the functional criterion than physicians without sport specialty $(71 \%) \mathrm{p}=0.0064, \mathrm{OR}=0.095$ $[0,017 ; 0,515]$. Sport physicians (64\%) selected significantly more a quantitative value to assess the pain (visual scale) than physicians without sport education $(41 \%) \mathrm{p}=0.03$. Few physicians use a questionnaire to assess the instability (less than $5 \%$ ).

Conclusions In daily practice, physicians with sport education are more aware of functional test guidelines to assess a LAS in RTP context. Few physicians use quantitative tools and measures to decide a safe RTP after a LAS.

\section{BALANCE CONTROL IN CHRONIC ANKLE INSTABILITY AND HEALTHY SUBJECTS: TRADITIONAL ANALYSIS OF CENTRE OF PRESSURE DISPLACEMENT AND NONLINEAR ANALYSIS OF ITS VARIABILITY}

\begin{abstract}
${ }^{1,2}$ José Esteves, ${ }^{2}$ Ricardo Dinis, ${ }^{3}$ Orlando Fernandes, ${ }^{4}$ Maria António Castro, ${ }^{2,5}$ Raul Oliveira, 2,5Pedro Pezarat Correia. ${ }^{1}$ Escola Superior de Saúde do Alcoitão, Lisboa, Portugal: ${ }^{2}$ Neuromuscular Research Lab, Faculdade de Motricidade Humana - Universidade de Lisboa, Lisboa, Portugal; ${ }^{3}$ Departamento de Desporto e Saúde - Universidade de Évora, Évora, Portugal; ${ }^{4}$ Escola Superior de Tecnologia da Saúde de Coimbra, Coimbra, Portugal; ${ }^{5}$ CIPER, Faculdade de Motricidade Humana - Universidade de Lisboa, Lisboa, Portugal
\end{abstract}

\subsection{6/bjsports-2021-IOC.98}

Background Many causes of chronic ankle instability (CAI) have been postulated and include deficits in proprioception, impaired neuromuscular firing patterns, reduced balance and postural control. Balance control can be studied with the traditional analysis of centre of pressure (CoP) displacement and nonlinear analysis of its variability.

Objective To compare balance control behaviour in CAI and healthy subjects, using the traditional linear and nonlinear variables for CoP displacement.

Design Comparative observational study.

Setting Laboratory.

Patients (or Participants) Students from 2 universities in Lisbon, who volunteered to participate in this study: 16 subjects with chronic ankle instability and 20 healthy subjects. Selection was based on the International Ankle Consortium (IAS) selection criteria position statement.

Interventions (or Assessment of Risk Factors) The independent variable was the presence of CAI according to IAS selection criteria.

Main Outcome Measurements CoP displacement was measured on a force plate during single leg stance for 60 seconds, on stable and unstable surfaces. Balance control was measured through traditional variables of CoP displacement: total displacement; medio-lateral and antero-posterior displacement; amplitude of medio-lateral and antero-posterior displacement. Variability of CoP displacement measured with nonlinear variables: sample entropy of medio-lateral and antero-posterior displacement; correlation dimension of medio-lateral and anteroposterior displacement.

Results On stable surface, no differences between groups for all the traditional variables were found but correlation dimension of CoP medio-lateral displacement had lower values on CAI group with statistical significance $(\mathrm{p}<0,05)$. On unstable surface no differences were found neither with traditional variables or variability nonlinear analysis.

Conclusions Besides no differences on most of variables, lower values of the correlated dimension of CoP displacement during one leg stance on a stable surface in CAI subjects may indicate a balance control system with less variability to adapt to the environment and the task demands. More studies are needed to continue the investigation about the balance control related to CAI. 